\title{
HABILIDADES Y CONOCIMIENTOS CONSTITUTIVOS DE LA ALFABETIZACIÓN TEMPRANA: SEMEJANZAS Y DIFERENCIAS SEGÚN EL ENTORNO SOCIAL Y LAS OPORTUNIDADES EDUCATIVAS*
}

\author{
EARLY LITERACY SKILLS: SIMILARITIES AND DIFFERENCES CAUSED BY SOCIAL \\ ENVIRONMENT AND SCHOOL OPPORTUNITIES
}

\author{
María Dolores Plana ${ }^{* *}$ y Julieta Fumagalli ${ }^{* * *}$
}

\begin{abstract}
*Trabajo realizado en el marco del Proyecto UBACyT F394 Acerca de la información semántica, morfológica y sintáctica. Evidencias psicolingüísticas y neurolingüísticas de las representaciones y acceso léxico.

**Doctora en Ciencias del Lenguaje. Investigadora del Proyecto UBACyT F394: Acerca de la información semántica, morfológica y sintáctica. Evidencias psicolingüísticas y neurolingüísticas de las representaciones y acceso léxico. E-Mail: doloresplana@gmail.com Amenábar 3544, Dpto. F, (C1429AEN) Ciudad Autónoma de Buenos Aires. República Argentina. ${ }^{* * *}$ Licenciada y Profesora en Letras. Becaria Doctoral del Consejo Nacional de Investigaciones Científicas y Técnicas (CONICET). Investigadora del Proyecto UBACyT F394: Acerca de la información semántica, morfológica y sintáctica. Evidencias psicolingüísticas y neurolingüísticas de las representaciones y acceso léxico. Ayudante de las Cátedras de Psicolingüística I y II en la Facultad de Filosofía y Letras de la Universidad de Buenos Aires (UBA).

E-Mail: julietafumigalli@yahoo.com
\end{abstract}

\section{RESUMEN}

Dada la relevancia del desarrollo temprano de los conocimientos o habilidades considerados precursores de la alfabetización, resulta fundamental, para la elaboración de propuestas pedagógicas, identificar aquellos aspectos en los que es necesario focalizar dichas propuestas. En este sentido, el estudio que se informa abordó la incidencia del medio en las habilidades tempranas de lectura y escritura en tres grupos de niños de diferente procedencia socioeconómica (niveles socioeconómicos medio urbano, bajo urbano y bajo rural). Los niños respondieron a 11 pruebas que evalúan las siguientes variables precursoras de la alfabetización: Habilidades de procesamiento fonológico, Conocimiento de las correspondencias, Habilidades de discriminación visual de letras y palabras, Habilidad de lectura de palabras en contexto, Capacidad de reconocimiento de acciones de lectura y escritura, Formas tempranas de escritura, Conocimiento de lenguaje técnico y Manejo de libro. Los resultados obtenidos señalan que existen diferencias entre los grupos en las tareas implicadas en el reconocimiento de lenguaje técnico, en las de conciencia fonológica y en las formas de escritura. Sin embargo, no se hallaron diferencias entre los niños de los distintos sectores socioeconómicos evaluados en las tareas de discriminación, manejo de libro y reconocimiento de acciones de lectura y escritura. En concordancia con el modelo evolutivo de Nelson (2007), los grupos muestran un desempeño similar en conocimientos que responden a procesos perceptivos básicos y difieren en aquellos que la investigación ha demostrado que requieren de la intervención de un adulto alfabetizado. 
Palabras clave: Lectura; Escritura; Habilidades perceptivas básicas; Precursores de la alfabetización; Nivel socioeconómico; Niños pequeños.

\section{ABSTRACT}

Due to the fact that early literacy skills are crucial for reading and writing learning process, it is important to identify them in order to design teaching activities. Most of the former literacy studies have been carried out with middle class children and show that reading and writing learning process begins very early at home. The rate and the way reading and writing are developed is highly related with parents educational level and the kind and frequency of the literacy activities they propose. There are differences between social groups with regard to these activities that may cause knowledge differences at early age that may explain reading and writing learning process failure in low income children.

From a developmental perspective there have been numerous studies focused mainly on literacy learning before formal teaching of reading and writing in school. These researches tempt to explore and describe the concepts, knowledge, skills and early actions of reading and writing as well as the social context where these developments occur.

In the framework of these observations, the aim of the present study was to compare early reading and writing skills in three groups of children from different socioeconomic environment. From theoretical and methodological point of view we have take in account a cognitive psychology perspective to explore those variables considered early literacy skills such as phonological awareness, early writing skills among others as well as we have adopted as teaching and learning process framework Vigotsky's zone of proximal development as well as Bruner's scaffolding.

The groups were described according to different variables such us age (measured up to May, 30th), sex and socioeconomic level. In order to measure the last variable, parents education level and job were considered according Sartú (1992) occupational scale. Using this variables three groups were selected: Group A was formed by 20 children mean age 5.5 -year-olds from middle urban income families who attended a public kindergarten school situated in a middle class neighborhood. Group B was formed by 19 kids mean age 5.3-year-olds from low income families who attended to a public kindergarten school in a low income urban neighborhood. Group C was formed by 17 children mean age 5.3-year-olds who attended a public kindergarten school from a low rural income area. Kids were assessed with 11 tasks: Phonological awareness, Letter-sound correspondences, Differences between letters, Words from pictures, Reading words in context, Recognition of writing and reading activities, Early reading and writing performance, Technical vocabulary knowledge, proper usage of books was tested.

The results of this study provides empirical information on the early literacy pattern of learning process of Spanish-speaking children as well as differences in knowledge and skills involved in this process between children from different socioeconomic groups.

Differences between the three groups were observed in technical vocabulary knowledge and phonological awareness tasks performance. There were no differences between socioeconomically groups in distinguishing letters and words from pictures, recognizing writing and reading activities and proper using of books. According to Nelson (2007), children have a similar performance in those activities based on basic cognitive skills and differ in the ones that involve adult's instruction. The similar performances of the groups tested seems to indicate that the knowledge involved in early literacy process is develop in a sequence characterized by an emerging knowledge about writing in the social environment. The fact that children did not grasp a complete competence in one knowledge before reaching it in other later ability shows that literacy acquisition is characterized by an overlapping development of different abilities and knowledge.

Key words: Reading; Writing; Early literacy skills; Basic perceptual skills: Social environment; Young children. 


\section{INTRODUCCIÓN}

El estudio de los procesos de lectura y escritura y de su aprendizaje ha tenido un importante desarrollo durante las últimas décadas en las que se perfilaron varias líneas de abordaje diferenciadas por el encuadre teórico, la metodología y el objeto de investigación.

Desde una perspectiva evolutiva se han realizado numerosos estudios centrados principalmente en aquellos desarrollos anteriores al aprendizaje formal de la lectura y la escritura en la escuela.

El objetivo del estudio que se informa precisamente fue indagar las conceptualizaciones, conocimientos, habilidades y acciones tempranas de lectura y escritura, así como el contexto social en el que estos desarrollos ocurren. Se considera que estos conocimientos y acciones pre-convencionales son una parte integral del aprendizaje de la lectura y de la escritura (Adams, 1990; Borzone de Manrique, 1997; Downing, 1970; Durkin, 1966; Ferreiro \& Teberosky, 1979; Landry \& Smith, 2006; Snow, Porche, Tabors \& Harris, 2007; Sulzby, 1983).

Como marco de los procesos de enseñanza y aprendizaje predominan el concepto de zona de desarrollo próximo, propuesto por Vigotsky (1979) y el de andamiaje de Bruner (1976), es decir, una perspectiva que enfatiza el contexto social del desarrollo y el aprendizaje en interacción. Sin embargo, se observan también algunas propuestas encuadradas en la teoría piagetiana como la de Ferreiro (Ferreiro \& Teberosky, 1979).

Para la realización del estudio que se informa se ha adoptado también la perspectiva teórica y metodológica de la Psicología Cognitiva con el fin de explorar las variables consideradas como precursoras de la alfabetización: Habilidades de procesamiento fonológico, Conocimiento de las correspondencias, Habilidades de discriminación visual de letras y palabras, Habilidad de lectura de palabras en contexto, Capacidad de reconocimiento de acciones de lectura y escritura, Formas tempranas de escritura y Conocimiento de lenguaje técnico -números, letras y palabras- (para una revisión ver Borzone de Manrique, 1997).

Asimismo se ha recurrido como modelo de referencia al planteo evolutivo de Nelson (1996, 2007) del niño experiencial en el que, en coincidencia con Vigotsky, se enfatiza la incidencia del medio pero se atiende también a las habilidades generales básicas que todos los niños poseen por su pertenencia a la especie humana (ver revisión en Plana, 2012). Para explicar la enseñanza y el aprendizaje, Nelson propone el concepto de construcción en colaboración, en el que se articulan los aspectos sociales e individuales de dichos procesos.

Los estudios sobre la alfabetización temprana, realizados en su mayoría con niños de nivel socioeconómico medio, señalan que este proceso se inicia muy tempranamente en el hogar y que su curso y ritmo de desarrollo están asociados al nivel de alfabetización de los padres y a la variedad y frecuencia de situaciones que promueven para el aprendizaje de los niños (Durkin, 1966; Newman, 2006; Whitehurst \& Lonigan, 1998; Yarden, Rowe \& MacGillivray, 2011).

Si bien existe consenso en admitir que los niños difieren entre sí en cuanto a sus conocimientos sobre la escritura y los usos del estilo de lenguaje escrito cuando ingresan a la escuela primaria, y que son los niños provenientes de sectores desaventajados los que han tenido un contacto menos frecuente con este instrumento de comunicación, la naturaleza y los alcances de estas diferencias no han sido objeto de un análisis sistemático, fundamentado en las investigaciones actuales sobre el proceso de alfabetización que comprenda todos los conocimientos y acciones de lectura y escritura implicados en dicho proceso (Lonigan, 2007; Phillips, Clancy-Menchetti \& Lonigan, 2008).

El objetivo de este estudio fue precisamente aportar evidencia empírica en relación a los postulados del modelo evolutivo de Nelson (1996), en la medida en que pretende identificar y describir comparativamente las habilidades y conocimientos mencionados anteriormente, que caracterizan las competencias iniciales del proceso de alfa- 
betización, en niños de diferente procedencia social. Se trata de identificar aquellos aspectos en los que niños de distinto nivel socioeconómico se asemejan y aquellos en los que se diferencian en tanto se considera que tendrán habilidades básicas similares y se diferenciarán en aquellos conocimientos y habilidades que dependen de la experiencia y la enseñanza que les proporciona el entorno social.

\section{ANTECEDENTES}

El estudio del proceso de adquisición de la lectura y la escritura en el niño antes de su ingreso a la escuela primaria tiene ya una larga tradición que puede remontarse a la década del 60, cuando Durkin realiza un trabajo pionero sobre los lectores precoces.

Paralelamente se comenzaron a analizar aspectos referidos a los conocimientos de los niños prelectores sobre la escritura: su interpretación de términos como letra, palabra, oración, sonido (Reid, 1966; Sinclair, Jarvella \& Levelt, 1978), el reconocimiento de acciones de lectura y escritura (Downing, 1970), las formas preconvencionales de escritura (Read, 1971) y la lectura y el reconocimiento de la lectura en el medio (Hiebert, Cioffi \& Antonak, 1984). Tanto estos como los primeros trabajos de Durkin (1966) revisten especial relevancia al focalizar la atención sobre lo que los niños saben antes de recibir instrucción formal, ya que sientan las bases para distinguir el inicio de la alfabetización convencional, sin dejar de valorar los conocimientos y las acciones anteriores a ese inicio como parte del proceso de alfabetización. Asimismo plantean nuevas hipótesis sobre factores claves de las experiencias tempranas que parecen fundamentales para los logros posteriores. Por ejemplo, el descubrimiento de la importancia de la conciencia fonológica como facilitadora del aprendizaje de la lectura y de la escritura, dado que el poder reconocer que las palabras están formadas por sonidos y que son esos sonidos los que las letras representan, promueve el aprendizaje de las correspondencias fonema - grafema
(Borzone de Manrique \& Gramigna, 1984; Ehri, Nunes, Willows, Schuster, YaghoubZadeh \& Shanahan, 2001; Elkonin, 1973; Liberman, Shankweiler, Fisher \& Carter, 1974; Storch \& Whitehurst, 2002).

Se diferencian en el proceso de alfabetización dos tipos de desarrollos: aquellos que tienen lugar como parte de un aprendizaje perceptivo resultado de la exposición a la escritura y aquellos en los que el adulto juega un rol fundamental como mediatizador de los aprendizajes (Borzone de Manrique, 1997). Esta diferenciación tiene importantes implicancias pedagógicas ya que asume que solo los conocimientos ligados al proceso de aprendizaje perceptivo (discriminación visual de letras y palabras) se adquieren por exposición a la escritura o inmersión en un medio con materiales escritos, mientras que la adquisición de todos los otros conocimientos y competencias, incluidos los relativos a los usos y estructuración discursiva del lenguaje oral, resulta de la intervención de un adulto competente en estas habilidades lingüísticas.

En este sentido, Gibson y Levin (1975) en un trabajo pionero observaron que los rasgos utilizados por los niños para la discriminación de letras eran muy similares a los utilizados por los adultos. En su contacto con la escritura, el niño no solo logra diferenciar las letras de las formas icónicas de los dibujos sino también identifica los rasgos críticos que las distinguen entre sí.

En otro trabajo fundacional Lavine (1972) observó que el ordenamiento lineal de la escritura jugaba un rol como rasgo crítico, pero no era un criterio suficiente cuando las unidades no eran letras. La variedad de las unidades fue un factor de mayor peso que mostró una tendencia a aumentar en importancia con la edad. Para los niños, la información relevante estaba en los rasgos de las letras, no en la forma global de la secuencia.

Por su parte, el análisis de los contextos en los cuales el niño experimenta con la escritura en el medio familiar muestra que en ellos la escritura cumple una variedad de funciones. Los diferentes usos y funciones que los niños experimentan con los adultos de su comuni- 
dad tienden a estar asociados con distintos tipos de textos e información (Murillo, Smith \& Schall, 2010; Sulzby \& Teale, 1991).

La participación en estos eventos les permite reconocer que la escritura tiene significado. Este reconocimiento puede tener lugar a edad temprana, alrededor de los 3 años y aún antes si el niño es alentado a atender a la escritura del medio como un sistema de señales que proporciona información (Spoelders \& van Damme, 1982).

Por otra parte, también se ha comprobado el valor predictivo de la conciencia fonológica y su relación causal con el aprendizaje (Anthony \& Francis, 2005; Bradley \& Bryant, 1985; Schneider et al., 1997). En uno de los primeros trabajos sobre la temática, Liberman y colaboradores (1974) compararon la habilidad de los niños de jardín de infantes (4 y 5 años) con la de niños de primer grado para contar fonemas y sílabas en palabras. Los resultados mostraron que la sílaba es una unidad de más fácil acceso que el fonema, como se había supuesto, dado que la sílaba es una unidad de producción mientras que los fonemas están codificados en la señal de habla. Estos resultados fueron replicados en varias lenguas: en sueco (Lundberg, Olofsson \& Wall, 1980), en español (Borzone de Manrique \& Gramigna, 1984), en francés (Morais, Cluytens \& Alegria, 1984), en italiano (Cossu, Shankweiler, Liberman, Tola \& Katyz, 1988). Todos estos trabajos coinciden en señalar que es necesario un mayor nivel de conciencia lingüística para analizar las palabras en fonemas que en sílabas y que los niños que concurren a primer grado aventajan a los niños de jardín de infantes en segmentación fonológica.

Desde estos primeros estudios hasta la actualidad se ha explorado en todas las lenguas de escritura alfabética la progresión en habilidades en conciencia fonológica según la tarea (reconocimiento, segmentación y síntesis, entre otras) y la unidad involucrada -rima, sílaba, ataque y coda, sonido inicial, sonido final- (e.g., Goikoetxea, 2005; Goswami \& Bryant, 1992; Jimenez \& Ortiz, 2000; Muter \& Snowling, 1998; Ziegler \& Goswami, 2005). Asimismo, se ha estudiado el efecto de estas habilidades a través de un meta-análisis que incorporó un número significativo de investigaciones en las que se encontró una fuerte incidencia de la conciencia fonológica en el aprendizaje de la lectura y de la escritura (Ehri et al., 2001).

El conocimiento del nombre de las letras ha sido investigado también como parte del proceso temprano de alfabetización. Chall (1967) observó que al inicio del aprendizaje este conocimiento era un buen predictor de los logros posteriores en lectura. $\mathrm{Si}$ bien la relevancia del nombre de las letras ha sido objeto de debate, estudios posteriores mostraron correlaciones altas entre el nombre y el sonido de la letra y la escritura (Stage \& Wagner, 1992, entre otros) y el valor predictivo de ese conocimiento (Bravo, Villalón \& Orellana, 2006; Diuk \& Ferroni, 2012). Se ha observado que el conocimiento de las letras en niños de jardín de infantes depende fuertemente de situaciones de enseñanza en el hogar (ver revisión en Diuk \& Ferroni, 2012).

A su vez, las formas tempranas de escritura fueron objeto de numerosos estudios que han demostrado que los niños escriben utilizando formas pre-convencionales de escritura mucho antes de hacerlo en forma convencional. En los primeros trabajos se atendió especialmente a la descripción de estas formas de escritura y a su desarrollo en relación con las características de linealidad y diferenciación de rasgos de la escritura convencional. Gibson y Levin (1975) encuentran que el desarrollo, que se inicia con las producciones gráficas en niños de alrededor de 18 meses, desemboca en la discriminación de rasgos gráficos de las letras como continuidad, intersección, linealidad y curvatura.

Por su parte, los modelos de aprendizaje de la lectura y de la escritura de palabras, sustentados en los últimos años por abundante evidencia empírica, han identificado una serie de estrategias, caracterizadas por el uso de determinados conocimientos y operaciones, que varían con el aprendizaje hacia estrategias más avanzadas (Ehri, 1999; Treiman, 1991). Cuando los niños leen una palabra a 
partir de pistas visuales salientes, como el logo de Coca Cola, o escriben su nombre de memoria porque han aprendido el trazado de las letras y su secuencia, se considera que recurren a una estrategia logográfica en la que no hay procesamiento analítico. El desarrollo de la conciencia fonológica y el conocimiento de las correspondencias grafema / fonema habilitan el uso de una estrategia analítica que implica el análisis de la palabra en los sonidos que la componen y la activación de las letras correspondientes. Esta estrategia analítica o alfabética puede ser parcial si solo representan algunos sonidos de las palabras, o completa, cuando representan todos los sonidos (Ehri, 1999).

La importancia de las primeras experiencias de alfabetización en el hogar para los aprendizajes de los niños y su desempeño escolar posterior en lectura y escritura y, muy especialmente, la relevancia del apoyo de los adultos que interactúan con los pequeños, ha recibido especial atención en el marco de los estudios sobre alfabetización temprana (Landry \& Smith, 2006; Ninio \& Bruner, 1978). Asimismo se han encontrado diferencias en las experiencias, los aprendizajes y en los logros escolares de niños provenientes de diferentes grupos sociales y culturales (e.g., Adams, 1990; Hammer, 2001).

Así por ejemplo, las estimaciones realizadas por Adams (1990) sobre los datos de Taylor (1983) y sus propios datos muestran diferencias importantes entre los niños con respecto a la cantidad de horas en las que tienen experiencias de alfabetización. En el caso de los niños de NSE medio, éstas ocupan alrededor de 4.000 horas antes de su ingreso a la escuela, mientras que entre los niños de NSE bajo se registran valores muy inferiores, de alrededor de 200 horas.

Dada la relevancia del desarrollo temprano de los conocimientos o habilidades considerados precursores de la alfabetización, resulta fundamental, para la elaboración de propuestas pedagógicas, identificar aquellos aspectos en los que es necesario focalizar dichas propuestas, en tanto, por proceder de un medio con un bajo nivel de alfabetización, los niños de NSE bajo pueden no haberlos adquirido.

Como ya se ha señalado, las diferencias en conocimientos que se establecen a edad temprana pueden dar cuenta, en parte, del fracaso en el aprendizaje de la lectura y la escritura que afecta mayoritariamente a los niños en situación de pobreza.

En el marco de estos planteos, se evaluó y comparó el desempeño de tres grupos de niños de 5 años, dos de ellos de NSE bajo y un grupo de NSE medio, en diferentes tareas con los que se exploró la adquisición de conocimientos precursores de la alfabetización.

\section{Metodología \\ SUJETOS}

Participaron de este estudio tres grupos de niños argentinos diferenciados por el sector socioeconómico de procedencia. Se describen los grupos en función de variables de base: edad, sexo e indicadores socioeconómicos y culturales tales como los niveles de escolaridad y ocupacional de los padres, evaluados con una escala de grupos ocupacionales (Sautú, 1992). Estos niños eran argentinos y alumnos de jardines de infantes públicos.

Grupo A: Este grupo estaba formado por 20 niños (11 varones y 9 mujeres), provenientes de familias de nivel socioeconómico medio, tenían una edad promedio de 5 años y 5 meses (extremos 5,0 y 5,10 ) y concurrían a la sala de 5 años de un jardín de jornada simple ubicado en un barrio residencial de la ciudad de Buenos Aires (Argentina).

Grupo B: Lo integraron 19 niños (13 varones y 6 mujeres), provenientes de familias de nivel socioeconómico bajo y tenían en promedio 5 años y 3 meses de edad (extremos 5,0 y 6,3 ) y eran alumnos de sala de 5 años de un jardín de infantes público de jornada completa ubicado en un barrio urbano marginado. Cabe señalar que un mes y medio antes de la admi- 
nistración de las pruebas, la maestra había comenzado a trabajar las habilidades de lectura y escritura de los niños a partir de los lineamientos del Programa ECOS (Borzone de Manrique \& Marro, 1990).

Grupo C: Este grupo estaba constituido por 17 niños ( 9 varones y 8 mujeres), provenientes de familias de nivel socioeconómico bajo. El promedio de edad era 5 años 3 meses (extremos 4,8 y 5,9). Los niños asistían a una sala unitaria de un jardín de infantes público de jornada simple ubicado en una zona rural. Es importante destacar que los niños de 5 años compartían las actividades con niños de 3 y 4 años.

\section{Materiales}

Se administraron 11 pruebas que varían según el grado de estructuración, algunas han sido específicamente diseñadas para este estudio y otras adaptadas al español o elaboradas a partir de criterios utilizados en otras investigaciones ${ }^{1}$.

1.- Acciones: Es una prueba de reconocimiento de acciones de lectura y escritura $\mathrm{y}$ tiene por objeto determinar si los niños pueden distinguir entre acciones de leer, escribir, dibujar y mirar. Consiste en una serie de cuatro fotos que muestran a personas realizando acciones de leer, pintar o dibujar, mirar y escribir. En dos de ellas se le pide a los niños que señalen a todas las personas que están leyendo y en las otras dos, a las personas que están escribiendo. Se computa el número de señalamientos correctos. Esta prueba fue adaptada de los subtests correspondientes al reconocimiento de acciones del Test of Linguistic

1 Los protocolos de estas pruebas pueden ser solicitados a las autoras.
Awareness de Ayers, Downing y Schaefer (1977).

2.- Contexto: Lectura de palabras en contexto. Esta prueba evalúa el reconocimiento de la escritura en el medio y el material consiste en ocho objetos familiares con etiquetas que portan escritura. Se le presenta al niño cada objeto o dibujo y se le pregunta: “QQué dice acá?”. Se registra la respuesta en forma literal. Luego se le solicita que señale dónde dice cada palabra y se registra si su respuesta es correcta.

3.- Manejo del libro: Esta prueba proporciona información sobre la diferenciación dibujo / escritura, direccionalidad de la escritura (izquierda / derecha; arriba / abajo) además de información sobre aspectos de manejo general del libro. El entrevistador presenta al niño un libro de cuentos, se lo entrega y realiza preguntas ("¿sabés qué es esto?, ¿dónde hay dibujos?, ¿dónde hay letras?, ¿dónde está escrito?"). Luego, el entrevistador lee el texto siguiendo con el dedo las palabras a medida que lee. Durante la lectura se detiene y hace nuevas preguntas ("vamos a leer, ¿por dónde comenzamos?, ¿por dónde seguimos?"). Las respuestas se computan como correctas cuando el niño: (a) reconoce el comienzo del libro, (b) reconoce la secuencia, (c) diferencia dibujo / escritura, (d) reconoce orientación izquierda / derecha y (e) reconoce la orientación arriba/abajo.

4.- Lenguaje técnico: En esta prueba se propone evaluar si el niño reconoce letras, palabras y números y si les asigna el nombre correcto. La prueba consiste en 12 series de estímulos en los que se alternan letras, números, garabatos, palabras, secuencias de letras y de números (Ayers, Downing \& Schaefer, 1977). En las tres primeras series, se le solicita al niño que señale los números; en las tres siguientes, las letras y en las seis restantes, las palabras. El entrevistador toma 
nota de todo los ítemes que señala el niño y se determina el porcentaje de ítemes correctos por serie. En caso de que el niño responda de manera azarosa, es decir señalando todos los ítemes de la serie sin tener en cuenta la consigna (señalar números, letras o palabras), no se contabilizan las respuestas del participante.

5.- Discriminación de letras: La discriminación visual de letras fue evaluada mediante una serie de 16 tarjetas en las que se presenta una letra en imprenta sobre cuatro letras alternativas. Antes de comenzar la prueba, se le muestran al niño tres tarjetas con ítemes de práctica para que vea en qué consiste la tarea. Luego se le pide que señale la letra superior (letra target) y que encuentre entre las otras letras presentadas, la que es igual. Se consigna el número total de ítemes señalados correctamente en la serie. Se toma también nota de las confusiones entre ítemes.

6.- Discriminación de palabras: La discriminación visual de palabras fue evaluada mediante dos series de 10 tarjetas. Una de ellas presenta las palabras escritas en letra imprenta minúscula y la otra, en imprenta mayúscula. Cada tarjeta presenta la palabra target en la parte superior y central, y cinco palabras alternativas debajo. Se siguió el mismo procedimiento que en la prueba de discriminación de letras.

7.- Sonido inicial: Es una prueba de apareamiento de palabras por sonido inicial que evalúa la habilidad para deslindar un sonido de una palabra de todos los demás. La tarea comprende 16 pares de palabras. El entrevistador presenta oralmente los pares y el niño debe responder si comienzan igual o no. Antes de comenzar la prueba el entrevistador explicita la tarea a través de tres ítemes de práctica: dice, prolongado el sonido inicial, dos palabras y señala que comienzan igual (o no). Le pide al niño que repita los ítemes de práctica y lo corrige si se equivoca. El puntaje de esta prueba se computa por el número de series (cuatro ítemes seguidos) que recibió una respuesta correcta. Se ha adoptado este criterio para evitar falsos aciertos o errores producto de una estrategia de respuesta al azar (puntaje 4/0).

8.- Segmentación fonológica: Es una prueba de segmentación de palabras en sonidos que evalúa la habilidad metalingüística del niño para manipular sonidos a través de la segmentación de palabras y el conteo del número de sonidos. Esta tarea es una adaptación al español realizada por Borzone de Manrique y Gramigna (1984) de la prueba clásica de Liberman y colaboradores (1974). La prueba consiste en 42 ítemes de uno, dos y tres segmentos distribuidos al azar en una lista. Todos los estímulos constituyen palabras monosílabas. La prueba se presenta como un juego de golpeteo en el que el entrevistador solicita a los niños que escuchen cada emisión e indiquen el número de sonidos mediante golpes dados con un lápiz sobre el escritorio. Si es necesario se corrigen las respuestas del niño. Antes de la aplicación se realizan ejercicios de entrenamiento. Esta prueba se evalúa de acuerdo a un criterio de segmentación: cuando el niño segmenta correctamente 6 ítemes consecutivos, se considera que respondió correctamente.

9.- Correspondencias: Para evaluar el conocimiento de los niños sobre las correspondencias sonido / letra se propuso una tarea de apareamiento: sonido inicial y la letra. La prueba consiste en 10 palabras que eran familiares para los niños. El entrevistador dice la palabra y le presenta una tarjeta con tres letras como opción y le pregunta "¿Cuál es la de...?”. Como práctica, el entrevistador escribe el nombre del niño a la vista de él, se lo lee y luego escribe tres letras y le pregunta cuál es la letra inicial de su nombre. El puntaje corresponde al número de apareamientos correctos realizados por el niño. 
10.- Secuencia: (Identificación de palabras en secuencia). Esta prueba evalúa el conocimiento del niño sobre la relación general entre habla y escritura, así como el hecho de que la escritura tiene significado, es lenguaje y lo que se dice se puede representar a través de las escritura. A la vista del niño el entrevistador escribe una oración ("Juan tiene una gata") y la lee señalando la escritura sin hacer pausas y con entonación normal. Luego se alternan preguntas del tipo: ¿Dónde dice...? ¿Acá qué dice? (señalando una palabra). Para administrar esta prueba se seleccionaron 9 ítemes de un trabajo realizado por Borzone de Manrique y Signorini (1988). Se computan las respuestas correctas.

11.- Escritura de palabras: Esta prueba tiene por objetivo conocer la forma de escritura usada por el niño en este tipo de tarea. El entrevistador solicita al niño que escriba su nombre y otras palabras que quiera escribir, también se le sugieren palabras. Se ha adoptado la descripción de las primeras formas realizada por Gibson y Levin (1975) para identificar las formas de escritura de los niños como pre-convencionales. Se consideró la copia como una forma particular. Por su parte, para las escrituras que guardan relación entre las letras y los sonidos, consideradas convencionales, se consideraron las estrategias planteadas por Ehri (1999): logográfica, analítica parcial y analítica completa.

\section{Procedimiento}

Las pruebas se aplicaron en entrevistas individuales semi-estructuradas. Antes de la aplicación, el entrevistador concurrió durante un mes dos veces por semana al aula y participó junto con los niños de diversas actividades con el objeto de familiarizarse con ellos.

La aplicación se realizó presentando las pruebas como un juego, por ejemplo, el juego de la letra perdida, el juego del golpeteo.
Se trató de crear un clima informal y cordial, aceptando que los niños preguntaran, contaran alguna experiencia, dibujaran o escribieran entre prueba y prueba. El número de pruebas a aplicar en cada entrevista dependía de la extensión de las mismas y la atención o fatiga que demostraba el niño. Las pruebas se aplicaron durante dos meses.

\section{RESULTADOS Y DISCUSIÓN}

Si se consideran los resultados de las pruebas en su conjunto se puede observar un patrón de desempeño para cada grupo. Los patrones presentan similitudes y diferencias entre los grupos que reflejan la relación entre los tipos de conocimientos y la interacción con el medio en el curso del proceso de alfabetización. Para describir estos patrones se ordenaron los puntajes obtenidos en las pruebas en forma decreciente, lo cual permite determinar la dificultad de acceso a cada tipo de conocimiento.

Como se muestra en el Gráfico 1, el patrón del Grupo A (nivel socioeconómico medio) es muy similar al del Grupo B (nivel socioeconómico bajo urbano), mientras que el patrón del Grupo C (nivel socioeconómico bajo rural) difiere del de los otros dos grupos, ya que los niños tienen mejor desempeño en la Prueba de Números y Letras que en la de Correspondencias.

Los valores más altos en los tres grupos corresponden a las pruebas de Discriminación, Manejo del libro y Reconocimiento de acciones. Es evidente que los niños con una mínima exposición a la escritura, observación y participación en acciones de lectura y escritura así como contacto con libros, pueden responder con eficacia a estas pruebas. Los valores medios para los grupos A y B corresponden a la prueba de Correspondencias. En esta última prueba el Grupo C, a diferencia de los otros dos, presenta valores muy bajos y no responde correctamente a ningún ítem de las pruebas Letras, Nombre de las letras y Sonido inicial.

Por su parte, los puntajes obtenidos por el Grupo B disminuyen abruptamente en rela- 
ción con los valores del Grupo A en las tres últimas pruebas: Sonido inicial, Nombre de las letras y Segmentación fonológica. Los conocimientos metalingüísticos que miden estas pruebas han sido señalados por diversas investigaciones como los que presentan una mayor asociación con el desempeño en lectura y escritura (e.g., Anthony \& Francis, 2005; Juel, Griffith \& Goung, 1986; Muter \& Snowling, 1998; Tunner \& Nesdale, 1985). Y es precisamente en estas pruebas en las que se observan mayores diferencias a favor del Grupo A. Asimismo, independientemente de las diferencias entre grupos, los conocimientos metalingüísticos son los de más difícil acceso como se observa en los resultados obtenidos en otras lenguas (Juel, Griffith \& Goung, 1986; Lomax \& Mc Gee, 1987).

En la Tabla 1 se muestran los puntajes promedio y desvíos estándar de cada prueba para los tres grupos. Como se puede observar, los valores del Grupo A son superiores a los de los grupos B y C en todas las medidas, en tanto que el Grupo $\mathrm{C}$ presenta valores inferiores. Para la consideración de estas diferencias es importante recordar que en el Grupo B se había comenzado a implementar el programa de alfabetización en forma sistemática un mes y medio antes de la aplicación de las pruebas y que el Grupo $\mathrm{C}$ pertenecía a una sala unitaria en la que los niños de 5 años compartían las actividades con niños de 3 y 4 años.

Los resultados obtenidos por los tres grupos en las pruebas de Discriminación de letras $[F(2,49)=2.779$; n.s. $]$ y en las de Discriminación de palabras [Palabras en mayúscula: $F(2,49)=.891$; n.s. y Palabras en minúscula: $F(2,49)=1.759$; n.s.] muestran los valores más altos y no indican diferencias significativas entre los grupos. Estas observaciones proporcionan nueva evidencia empírica con respecto a la naturaleza de este aprendizaje perceptivo que requiere de un mínimo de contacto con la escritura, tal como fue planteado en trabajos anteriores (Borzone de Manrique, 1994; Gibson \& Levin, 1975).

Asimismo, si se desglosan los puntajes de la prueba Manejo del libro, como se mues- tra en la Tabla 2, se observa que todos los niños pudieron diferenciar la escritura del dibujo, lo que indica que este conocimiento es parte del proceso de aprendizaje perceptivo.

Sin embargo, si se comparan los puntajes obtenidos en las pruebas de Discriminación y Dibujo / Escritura con los de Orientación, se comprueba que dentro de los conocimientos gráficos, el reconocimiento de la orientación de la escritura presenta valores inferiores con respecto a los otros, lo que corrobora la diferencia entre el aprendizaje perceptivo por mera exposición a la escritura, que da lugar a una progresiva especificidad en la discriminación de los estímulos, del proceso de aprendizaje de todos los demás conocimientos, que, por ser convencionales, requieren de la intervención de un adulto alfabetizado que realice señalamientos explícitos.

Para analizar los resultados obtenidos en las pruebas que evalúan Manejo del libro, Reconocimiento de acciones, Lectura de contexto y Lenguaje técnico se realizó un análisis que no detectó diferencias significativas entre los grupos al comparar Manejo del libro $[F(2,49)=.544$; n.s.], Reconocimiento de acciones $[F(2,49)=.281 ;$ n.s. $]$ y Lectura de contexto $[F(2,49)=1.575$; n.s.]. El mismo análisis se aplicó para comparar los resultados alcanzados por los grupos en las pruebas que evalúan Lenguaje técnico. En esta oportunidad no se encontraron diferencias significativas entre los grupos A y $\mathrm{B}$, pero sí entre estos grupos y el C en Números $[F(2,49)=4.552 ; p<.01]$, Letras $[F(2,49)=4.869 ; p<.01]$ y Palabras $[F(2$, $49)=5.911 ; p<.001]$.

En el caso de las pruebas de Lenguaje técnico, los puntajes más altos fueron en la Prueba de Palabras en los tres grupos. Puede pensarse que los niños asocian el término palabra con la escritura y distinguen las palabras de los otros estímulos de la prueba en base a las características perceptivas que les permiten identificar la escritura, esto es la multiplicidad y variedad de caracteres (Lavine, 1972).

Por su parte, la Prueba de Lectura de contexto reveló puntajes bajos en los tres 
grupos. Sin embargo, los comentarios de los niños y el hecho de que nombren el producto, señalando la escritura, parece indicar que reconocen que tiene significado.

En algunos trabajos evolutivos (Lomax $\&$ Mc Gee, 1987; Mason, 1980) se ha atribuido a la lectura de carteles y logos un papel relevante como parte del proceso de aprendizaje de la lectura y la escritura. Se piensa que la lectura del contexto refleja el reconocimiento del niño de que los objetos y el habla pueden ser representados en la escritura. Asimismo, se considera que esta forma de lectura basada en pistas visuales perceptivamente salientes constituye una de las primeras fases, la fase o estrategia logográfica, de lectura de palabras (Ehri, 1999).

Si bien la lectura de contexto es relevante en tanto manifiesta dicho reconocimiento, no parece desempeñar un papel fundamental en la alfabetización temprana dado que los niños del Grupo A, cuyos puntajes son bajos en esta tarea, obtuvieron puntajes más altos en Conocimiento de las correspondencias, lo que es fundamental para aprender a leer y a escribir (para una revisión ver Adams, 1990).

Los resultados de la Prueba de Sonido inicial que evalúa habilidades metalingüísticas muestran valores muy bajos en los tres grupos. El análisis de variancia aplicado a estos datos indica que hay una diferencia significativa entre ellos $[F(2,49)=4.989$; $p<.01]$. El análisis post hoc de contraste Bonferroni no muestra diferencias entre los grupos A y B, pero señala que el desempeño de ambos grupos es significativamente diferente al del Grupo $\mathrm{C}(p<.001)$ a favor del Grupo A $(p<.01)$ y a favor del Grupo B.

Si se atiende a los desvíos estándar de la tarea de Sonido inicial, se observan en todos los grupos valores muy altos, lo que indica gran variación intra-grupo. En el caso del Grupo A, cuyos valores son superiores a los de los otros dos grupos, estos resultados podrían dar cuenta, junto con los valores obtenidos en Correspondencias, de su desempeño en Escritura. Como se muestra en la Tabla 3, más de un 50\% de los niños de este grupo escribieron palabras en forma con- vencional, en coincidencia con numerosos trabajos que señalan la estrecha relación entre las habilidades de sensibilidad fonólogica y las de escritura (Anthony, Williams, Aghara, Dunkelberger \& Novak, 2010; Treiman, 1991).

Por su parte, la tarea de Segmentación de palabras en sonidos presentó una seria dificultad para todos los niños y mostró ser una habilidad más compleja que deslindar el sonido inicial de una palabra, como se ha observado en otros estudios (Anthony, Lonigan, Driscoll, Phillips \& Burgess, 2003; Liberman et al., 1974).

Los valores obtenidos en la prueba de identificación de palabras en Secuencia muestran un desempeño similar en los tres grupos que presentan porcentajes de respuesta cercanos al 50\%. El análisis de variancia no acusa diferencias significativas entre ellos $[F(2,49)=2.553$; n.s. $]$. Es posible pensar que por sus características, esta prueba da lugar a cierto aprendizaje en el curso de su aplicación. En efecto, el entrevistador antes de pedir la identificación de cada palabra, repite la lectura de la oración escrita ante la vista del niño. Como se trata de una oración de pocas palabras, el niño puede retenerla en su memoria, repetirla mentalmente y utilizar la representación que mantiene en la memoria operativa para realizar las correspondencias con la secuencia de palabras escritas.

Los resultados de las pruebas correspondientes al Nombre de las letras presentan valores muy bajos en todos los grupos. El análisis de variancia muestra que existe una diferencia significativa entre los grupos [Letras mayúsculas: $F(2,49)=8.162$; $p<.001]$ y Letras minúsculas: $[F(2$, $49)=5.717 ; p<.001]$. El análisis post hoc de contraste Bonferroni señala que existen diferencias entre el Grupo A y el Grupo B tanto en Letra mayúscula $(p<.001)$ como en Letra minúscula $(p<.01)$ a favor del Grupo A. No se incluyó en este análisis al Grupo C por presentar un porcentaje cero. La relevancia de las diferencias entre grupos puede ponderarse mejor si se atiende a resultados de estudios recientes (Diuk \& Ferroni, 2012) en 
los que se encuentra que los niños que tienen un mejor desempeño en lectura son también los que conocen un mayor número de letras.

Es interesante señalar que el porcentaje de aciertos obtenidos para el inglés (Lomax \& Mac Gee, 1987) en pruebas similares aplicadas a niños de 5 años, es mucho más alto $(92 \%)$ que los obtenidos en este estudio para el español. Las diferencias podrían deberse a la modalidad de las actividades que proponen los adultos que introducen a los niños tempranamente en estos conocimientos, dadas las características del sistema ortográfico del inglés que, a diferencia del español, presenta una ortografía opaca.

En la Prueba de Correspondencias sonido-letra los resultados obtenidos arrojan diferencias significativas entre los grupos $[F(2,49)=16.448 ; p<.001]$. El análisis post hoc señala que existen diferencias entre los grupos A y B $(p<.05)$; A y C $(p<.001)$ $\mathrm{y}$ entre B y C $(p<.001)$

El hecho de que el desempeño de todos los grupos sea superior en la tarea de Correspondencias que la del Nombre de las letras puede deberse, por un lado, a las características ortográficas y fonéticas del español (se trata de una lengua transparente en la cual el nombre de las vocales y de varias consonantes corresponde a los sonidos) y, por otro, a las características de la tarea. En el caso de Correspondencias se le proporcionan al niño los dos elementos, la letra y el sonido, ya que se pronuncian las palabras opcionales. Por el contrario, cuando se presentan las letras, el niño tiene un solo elemento, la forma gráfica, para activar el nombre requerido.

En la Tabla 3 se presentan los porcentajes de niños que escribieron palabras en las diversas formas de escritura consideradas. En los grupos B y C, un porcentaje de niños se negó a escribir palabras: $21 \%$ y $35 \%$, respectivamente.

Los resultados de las pruebas de Escritura muestran diferencias muy marcadas entre los tres grupos en cuanto a la variedad y tipos de forma de escritura. En efecto, mientras que más del 50\% de los niños del Grupo A muestra en su escritura un principio de reconocimiento de la relación entre la escritura alfa- bética y la estructura fonológica de las palabras que aquella representa, ningún niño de los otros dos grupos parece tener este conocimiento. La escritura de los niños de los grupos $\mathrm{B}$ y $\mathrm{C}$ corresponde a formas menos evolucionadas. Dentro de éstas, el Grupo B presenta un rango mayor de variación (seis formas distintas) que el otro grupo (cuatro formas). La diferencia con el Grupo C podría deberse al contacto frecuente con la escritura que tenía lugar en la experiencia de alfabetización de la que participan los niños del Grupo B.

En el Grupo A más del $50 \%$ de los niños escribe palabras en forma convencional y su nombre, solamente un $26 \%$ escribe sólo el nombre. El 20\% restante produce formas en las que las letras que utilizan no se corresponden con los sonidos de las palabras que dicen escribir. Sin embargo demuestran reconocimiento de aspectos de la escritura como la forma y linealidad de la secuencia de letras.

Solo dos niños del Grupo B utilizaron una estrategia de escritura logográfica: escribieron papá y mamá. Ninguno supo escribir su nombre $\mathrm{y}$, aunque producen una variedad de formas de escritura, un alto porcentaje reproduce palabras presentes en el aula como la fecha, canciones y rimas que conocen y leen junto con la maestra. Entre la variedad de formas de escritura realizadas por los niños de este grupo, la copia, a la que recurre un porcentaje importante $(36.8 \%)$, parece ser una vía de explorar la forma de las letras, su nombre y otras características de la escritura (linealidad de elementos).

Con respecto al Grupo C, sólo un niño utiliza una estrategia logográfica, escribe papá y mamá. Todos se niegan a leer lo que han escrito manifestando que no saben o que no dice nada, son letras.

\section{DisCUSIÓN GENERAL}

Los resultados obtenidos en el estudio realizado proporcionan información empírica sobre las características del proceso de alfabetización temprana en niños de habla 
española, así como sobre las diferencias en los conocimientos y habilidades involucradas en este proceso entre niños de distinta procedencia socioeconómica.

La similitud global de los patrones de desempeño en las pruebas parece indicar que los conocimientos implicados en el proceso de alfabetización se desarrollan y adquieren en una secuencia caracterizada por un dominio temprano de los conocimientos referidos a la escritura y conocimientos incipientes sobre el sistema de escritura. Dentro de estos, el aprendizaje de las correspondencias letra / sonido y alguna forma de conciencia fonológica, como la que refleja la prueba de sonido inicial, parece anterior al dominio de las habilidades analíticas implicadas en la segmentación de palabras y manipulación de fonemas.

Por otra parte, que los niños no alcancen un dominio completo de un conocimiento antes de adquirir cierta habilidad en otro posterior de la secuencia parece mostrar que la relación entre conocimientos es la de facilitar y promover el desarrollo de los siguientes. Los datos proporcionados por diversos estudios longitudinales dan apoyo a esta hipótesis (Hiebert et al., 1984; Lomax \& Mc Gee, 1987) desde la que se visualiza el proceso de alfabetización como la adquisición en paralelo de conocimientos y estrategias que dan lugar a acciones pre-convencionales de lectura y escritura.

La secuencia de desarrollo descripta presenta una similitud global en español y en inglés (Hiebert et al., 1984; Lomax \& Mc Gee, 1987; Mason, 1980) a pesar de la diferencia señalada en el caso del nombre de las letras.

Con respecto a la diferencia de desempeño entre los tres grupos que participaron en el estudio, si se consideran los puntajes obtenidos en el marco de un modelo evolutivo que asume cierta secuencia de desarro1lo, puede decirse que los niños del Grupo A han alcanzado niveles más avanzados de desarrollo que los niños de los grupos B y C. En efecto, mientras que todos los niños tienen en general un buen desempeño en las pruebas que evalúan Discriminación de palabras y letras, Manejo del libro y Reconocimiento de acciones, los niños del Grupo A aventajan a los otros dos grupos en el conocimiento metalingüístico y conocimiento de las correspondencias entre el habla y la escritura.

El hecho de que los grupos tengan un desempeño similar en conocimientos que responden a procesos perceptivos básicos y difieran en aquellos que la investigación ha mostrado que requieren de la intervención de un adulto alfabetizado, es congruente con el modelo evolutivo de Nelson (2007). Asimismo, da cuenta, en parte, del fracaso escolar que experimentan mayoritariamente los niños de sectores desfavorecidos, en tanto ingresan a la enseñanza formal sin conocimientos fundamentales para la alfabetización y su avance, como lo predice el efecto Mateo (Diuk \& Ferroni, 2012, Stanovich, 1986) resulta difícil y costoso. Este trabajo permite identificar y cuantificar la brecha existente entre los niños de distinta procedencia socioeconómica, al mismo tiempo que reafirma el peso de los aprendizajes tempranos en el hogar y la importancia de la escolarización inicial, sobre todo en aquellos niños de ámbitos socio-económicamente desventajados.

De hecho, investigaciones realizadas en nuestro medio aportaron evidencia acerca del potencial de los niños para aprender, dada una intervención pedagógica apropiada (Diuk, Borzone \& Rosemberg, 2000). Sin embargo, también se encontró evidencia de que la falta de oportunidades tempranas para el aprendizaje de la lectura y la escritura tiene consecuencias tanto en el ritmo de adquisición, que se encuentra lentificado (Diuk et al., 2003) como en el patrón de aprendizaje (Borzone \& Diuk, 2001). 
Plana y Fumagalli

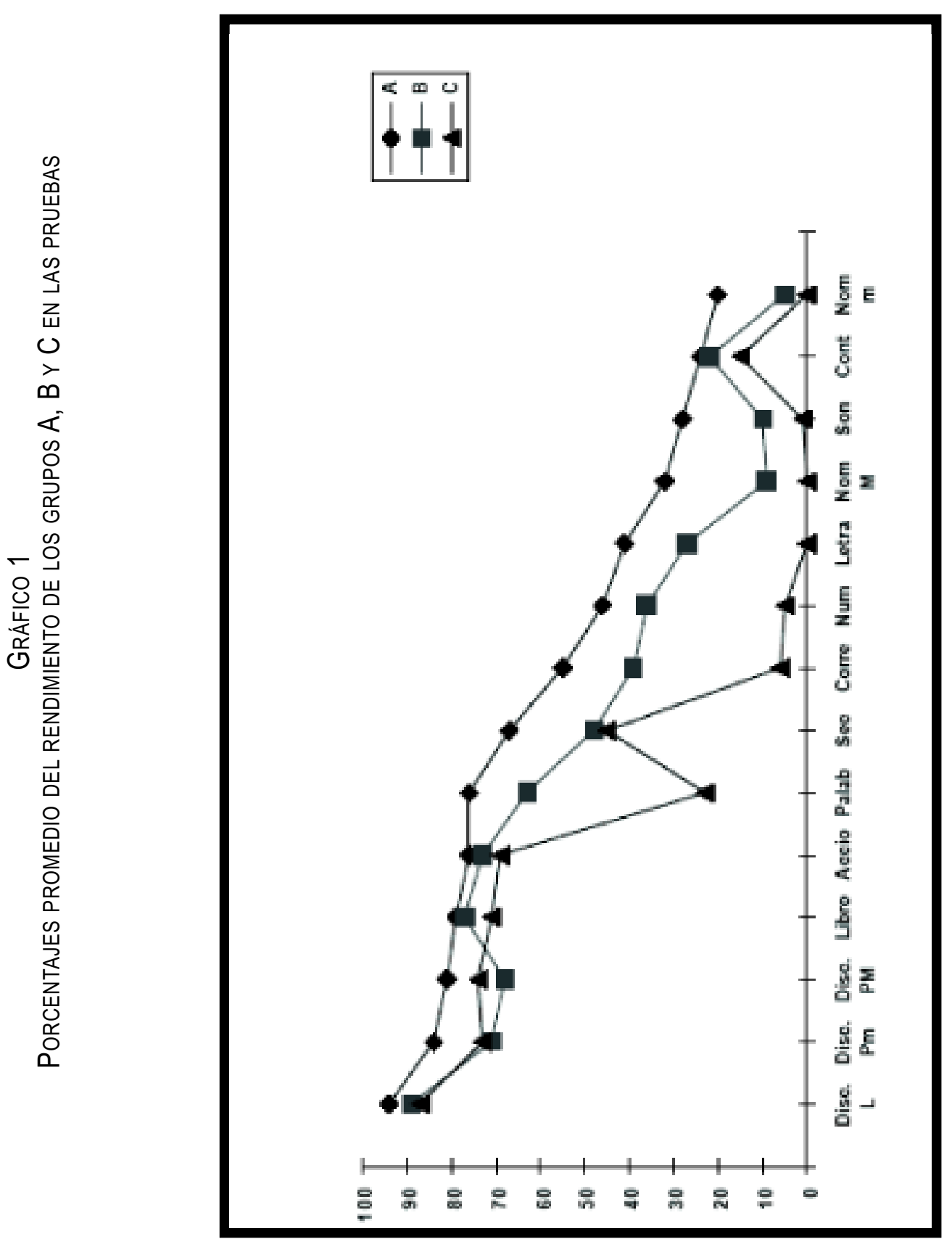


Habilidades y conocimientos constitutivos de la alfabetización temprana

TABLA 1

PORCENTAJES PROMEDIO Y DESVIOOS ESTÁNDAR DE LOS PUNTAJES OBTENIDOS EN LAS PRUEBAS SEGÚN GRUPO

\begin{tabular}{|c|c|c|c|c|c|c|}
\hline Pruebas & $M^{\mathrm{G}}$ & $\mathrm{A}$ & $M^{\mathrm{G}}$ & ${ }^{\mathrm{O}} \mathrm{B}$ & $M^{\mathrm{Gr}}$ & $\begin{array}{l}\mathrm{O} C \\
D E\end{array}$ \\
\hline $\begin{array}{l}\text { Conceptos sobre la escritura } \\
\text { Libro } \\
\text { Acciones } \\
\text { Contexto }\end{array}$ & $\begin{array}{r}79.3 \\
76.25 \\
24.75\end{array}$ & $\begin{array}{c}23 \\
25 \\
16.1\end{array}$ & $\begin{array}{l}77.35 \\
73.68 \\
22.63\end{array}$ & $\begin{array}{l}23.54 \\
29.43 \\
15.93\end{array}$ & $\begin{array}{l}71.33 \\
69.23 \\
15.38\end{array}$ & $\begin{array}{l}16.54 \\
23.17 \\
11.98\end{array}$ \\
\hline $\begin{array}{l}\text { Lenguaje técnico } \\
\text { Números } \\
\text { Letras* } \\
\text { Palabras }\end{array}$ & $\begin{array}{l}46.5 \\
41.6 \\
76.6\end{array}$ & $\begin{array}{c}45 \\
47 \\
39.2\end{array}$ & $\begin{array}{l}36.68 \\
27.94 \\
63.15\end{array}$ & $\begin{array}{l}42.78 \\
38.82 \\
49.55\end{array}$ & $\begin{array}{c}5.07 \\
0 \\
23.07\end{array}$ & $\begin{array}{r}18.3 \\
43.85\end{array}$ \\
\hline $\begin{array}{l}\text { Conocimientos gráficos } \\
\text { Discriminación de letras } \\
\text { Discrimin. de palabras en mayúscula } \\
\text { Discrimin. de palabras en minúscula }\end{array}$ & $\begin{array}{r}94.04 \\
84.5 \\
81.5\end{array}$ & $\begin{array}{c}6.26 \\
18.8 \\
18.5\end{array}$ & $\begin{array}{l}89.77 \\
71.57 \\
68.94\end{array}$ & $\begin{array}{c}8.04 \\
31.13 \\
39.28\end{array}$ & $\begin{array}{l}87.69 \\
73.07 \\
74.61\end{array}$ & $\begin{array}{r}9.62 \\
13.15 \\
24.7\end{array}$ \\
\hline $\begin{array}{l}\text { Conciencia fonológica } \\
\text { Sonido inicial } \\
\text { Segmentación en sonidos* }\end{array}$ & $\begin{array}{c}28.75 \\
15\end{array}$ & $\begin{array}{l}33.7 \\
6.25\end{array}$ & $\begin{array}{c}10.52 \\
0\end{array}$ & 22.53 & $\begin{array}{c}1.92 \\
0\end{array}$ & 6.93 \\
\hline $\begin{array}{l}\text { Conocimiento de las correspondencias } \\
\text { Secuencias } \\
\text { Nombre de letras minúsculas } \\
\text { Nombre de letras mayúsculas* } \\
\text { Letra / Sonido }\end{array}$ & $\begin{array}{l}67.56 \\
20.88 \\
32.2 \\
55.5\end{array}$ & $\begin{array}{l}31 \\
31.9 \\
31.9 \\
28\end{array}$ & $\begin{array}{c}48.97 \\
5.22 \\
9.1 \\
39.47\end{array}$ & $\begin{array}{r}32.81 \\
15.49 \\
22.55 \\
23.91\end{array}$ & $\begin{array}{c}45.98 \\
0 \\
0 \\
6.92\end{array}$ & 14.93 \\
\hline
\end{tabular}

${ }^{*}$ Porcentaje que alcanza criterio 


\section{TABLA 2}

PoRCENTAJES PROMEDIO Y DESVIOOS ESTÁNDAR DE LOS PUNTAJES OBTENIDOS EN LA PRUEBA MANEJO DEL LIBRO DISCRIMINADOS POR ÍTEMES

\begin{tabular}{|c|c|c|c|c|c|c|}
\hline \multirow[b]{2}{*}{ Pruebas } & \multicolumn{2}{|c|}{ Grupo A } & \multicolumn{2}{|c|}{ Grupo B } & \multicolumn{2}{|c|}{ Grupo C } \\
\hline & $M$ & $D E$ & $M$ & $D E$ & $M$ & $D E$ \\
\hline Libro & 94.90 & 12.45 & 91.10 & 18.93 & 81.84 & 29.35 \\
\hline Dibujo / Escritura & 100 & & 100 & & 100 & \\
\hline Orientación & 66.40 & 36.26 & 50.73 & 43.53 & 38.30 & 38.14 \\
\hline
\end{tabular}

TABLA 3

PORCENTAJES DE LAS FORMAS DE ESCRITURA UTILIZADAS POR LOS NIÑOS DE LOS TRES GRUPOS

\begin{tabular}{|c|c|c|c|c|}
\hline \multicolumn{2}{|c|}{ Pruebas } & Grupo A & Grupo B & Grupo C \\
\hline \multirow{2}{*}{ Escrituras pre-convencionales } & Secuencias de grafemas o signos & 20.9 & 36.6 & 40.6 \\
\hline & Copia & 0 & 36.8 & 0 \\
\hline \multirow{4}{*}{ Escrituras convencionales } & Logográfica & 0 & 5.2 & 1.6 \\
\hline & Analítica parcial & 5.2 & 0 & 0 \\
\hline & Analítica completa & 47.3 & 0 & 0 \\
\hline & Nombre & 26.3 & 0 & 11.6 \\
\hline Se niegan & & 0 & 21 & 35 \\
\hline
\end{tabular}




\section{REFERENCIAS BIBLIOGRÁFICAS}

Adams, M. (1990). Beginning to read. Thinking and learning about print. Cambridge, MA: The MIT Press.

Anthony, J. \& Francis, D. (2005). Development of phonological awareness. Current Directions in Psychological Science, 14(5), 255-259. doi: 10.1111/j.0963-7214.2005.0037 6.x.

Anthony, J.L., Lonigan, C.J., Driscoll, K., Phillips, B.M. \& Burgess, S.R. (2003). Phonological sensitivity: A quasi-parallel progression of word structure units and cognitive operations. Reading Research Quarterly, 38, 470-487. doi: 10.1598/ RRQ. 38.4.3.

Anthony, J.L., Williams, J.M., Aghara, R.G., Dunkelberger, M.J. \& Novak, B. (2010). Assessment of individual differences in phonological representation. Reading and Writing, 23(8), 969-994. doi: 10.1007/s11145-09-918 57.

Ayers, I.H., Downing, J. \& Schaefer, B. (1977). Test of linguistic awareness in reading readiness. Victoria, Canadá: University of Victoria.

Borzone de Manrique, A.M. (1994). Leer y escribir a los 5 [Reading and writing at 5 years old]. Buenos Aires: Aique.

Borzone de Manrique, A.M. (1997). Los procesos de enseñanza y aprendizaje inicial de la lectura y la escritura: Diferencias socioculturales [Early literacy teaching and learning processes: Sociocultural differences]. Tesis Doctoral no publicada. Universidad de Buenos Aires. Buenos Aires, Argentina.

Borzone, A.M. \& Diuk, B. (2001). El aprendizaje de la escritura en español: Estudio comparativo entre niños de distinta procedencia social [Learning to write in Spanish: A comparative study between children from different social environment]. Interdisciplinaria, 18(1), 1-33.

Borzone de Manrique, A.M. \& Gramigna, S. (1984). La segmentación fonológica y silá- bica en niños de preescolar y primer grado [Phonological and syllabic segmentation in kindergarten and first grade children]. Lectura y Vida, 5, 4-13.

Borzone de Manrique, A.M. \& Marro, M. (1990). Lectura y escritura: Nuevas propuestas desde la investigación y la práctica [Reading and writing: new approaches from researching and practice]. Buenos Aires: Kapelusz.

Borzone de Manrique, A.M. \& Signorini, A. (1988). Del habla a la escritura: La conciencia lingüística como una forma de transición natural [From talking to writing: linguistic awareness as a natural transition]. Lectura y Vida, 9 , 5-9.

Bradley, L. \& Bryant, P. (1985). Rhyme and reason in reading and spelling. Ann Arbor: University of Michigan Press.

Bravo, L., Villalón, M. \& Orellana, E. (2006). Diferencias en la predictibilidad de la lectura entre primer año y cuarto año básicos [Differences in reading predictability between first and fourth grade]. Psykhe, 15(1), 3-11.

Bruner, J.S. (1976). Learning how to do things with words. Trabajo presentado en el Wolfson Lecture. Wolfson College, Oxford.

Chall, J.S. (1967). Learning to read: The great debate. Nueva York: McGraw-Hill.

Cossu, G., Shankweiler, D., Liberman, I.Y., Tola, G. \& Katyz, L. (1988). Awareness of phonological segment and reading ability in Italian children. Applied Psychology, 9, 1-16. doi: $10.1017 / \mathrm{S} 0142716400000424$.

Diuk, B., Borzone, A.M. \& Rosemberg, C.R. (2000). El fracaso escolar entre los niños de sectores pobres: Una alternativa pedagógica intercultural [School failure among poor children: An intercultural educational alternative]. C\&E: Cultura y Educación, 12(12), 23-33. doi: 10.1174/113564000753837089.

Diuk, B. \& Ferroni, M. (2012). Dificultades lectoras en contextos de pobreza: ¿Un caso de Efecto Mateo? [Reading difficulties in pover- 
ty contexts: A Mattew Effect case?]. Revista Psicologia Escolar e Educacional, Psicologia Escolar e Educacional, 16(2), 209-217. doi: 10.1590/S1413-85572012000200003.

Diuk, B., Signorini, A. \& Borzone, A.M. (2003). Las estrategias tempranas de lectura de palabras en niños de 1er. ciclo de E.G.B.: Un estudio comparativo entre niños procedentes de distintos sectores sociales [Early reading strategies in first grade children: A comparative study with children from different social environments]. Psykhe, 12(2), 51-62.

Downing, J. (1970). Children's concepts of language in learning to read. Educational Research, 12(2), 106-112. doi: 10.1080/001318 8700120203.

Durkin, D. (1966). Children who read early. New York: Teachers College Press.

Ehri, L.C. (1999). Phases of development in learning to read words. En J. Oakhill \& R. Beard (Eds.), Reading development and the teaching of reading. A psychological perspective (pp. 79-108). Oxford, England: Blackwell.

Ehri, L.C., Nunes, R.S., Willows, D.M., Schuster, B., Yaghoub-Zadeh, Z. \& Shanahan, T. (2001). Phonemic awareness instruction helps children learn to read: Evidence from the National Reading Panel's meta-analysis. Reading Research Quarterly, 36, 250-287.

Elkonin, D.B. (1973). USSR. En J. Downing (Ed.), Comparative reading: Cross-national studies of behavior and processes in reading and writing (pp.137-172). New York: Macmillan.

Ferreiro, E. \& Teberosky, A. (1979). Los procesos de escritura en el desarrollo del niño [Writing processes in children development]. Buenos Aires: Siglo XXI.

Gibson, E. \& Levin, H. (1975). The psychology of reading. Cambridge, Massachusets: The MIT Press.

Goikoetxea, E. (2005). Levels of phonological awareness in preliterate and literate Spanish- speaking children. Reading and Writing, 18, 51-79. doi: 10.1007/s11145-004-1955-7.

Goswami, U. \& Bryant, P.E. (1992). Rhyme, analogy, and children's reading. En P.B. Gough, L.C. Ehri \& R. Treiman (Eds.), Reading acquisition (pp. 49-62). Hillsdale, NJ: Erlbaum.

Hammer, C.S. (2001). Come, sit down and let mamma read: Book reading interactions between African American mothers and their infants. En J. Harris, A. Kamhi \& K. Pollock (Eds.), Literacy in African American communities (pp. 21-44). Hillsdale, NJ: Erlbaum.

Hiebert, E., Cioffi, G. \& Antonak, R.F. (1984). A developmental sequence in preschool children's acquisition of reading readiness skills and print awareness concepts. Journal of Applied Developmental Psychology, 5(2), 115126. doi: 10.1016/0193-3973(84)90012-1.

Jimenez, J.E. \& Ortiz, M.R. (2000). Metalinguistic awareness and reading acquisition in the Spanish Language. The Spanish Journal of Psychology, 3, 37-46.

Juel, C., Griffith, P.L. \& Goung, P.B. (1986). Acquisition of literacy: A longitudinal study of children in first and second grade. Journal of Educational Psychology, 78(4), 243-255. doi: 10.1037/0022-0663.78.4.243.

Landry, S.H. \& Smith, K.E. (2006). The influence of parenting on emerging literacy skills. En D.K. Dickinson \& S.B. Neuman (Eds.), Handbook of early literacy research, Vol. 2 (pp. 135-148). New York, NY: Guilford.

Lavine, L.O. (1972). The development of perception of writing in pre-reading children: A cross-cultural study. New York: Department of Human Development, Cornell University.

Liberman, I., Shankweiler, D., Fisher, F. \& Carter, B. (1974). Explicit syllable and phonemic segmentation in the young child. Journal of Experimental Child Psychology, 18(2), 201-212. doi: 10.1016/0022-0965(74) 90101-5. 
Lomax, R. \& Mc Gee, L. (1987). Young children's concepts about print and reading: Toward a model of word reading acquisition. Reading Research Quarterly, 22, 237-256.

Lonigan, C.J. (2007). Vocabulary development and the development of phonological awareness skills in preschool children. En R.K. Wagner, A.E. Muse \& K.R. Tannenbaum (Eds.), Vocabulary acquisition: Implications for reading comprehension (pp. 15-31). New York: Guilford. doi: 10.2307/747667.

Lundberg, I., Olofsson, A. \& Wall, S. (1980). Reading and spelling skills in the first school years predicted from phoneme awareness skills in kindergarten. Scandinavian Journal of Psychology, 21, 159-173. doi: 10.1111/j. 1467-9450.1980.tb00356.x.

Mason, J.M. (1980). When do children begin to read: An exploration of four-year-old children's letter and word competencies. Reading Research Quarterly, 15, 203-227. doi: 10.23 $07 / 747325$.

Morais, J., Cluytens, M. \& Alegria, J. (1984). Segmentation abilities of dyslexics and normal readers. Perceptual and Motor Skills, 58, 221-222. doi: 10.2466/PMS.58.1.221-222.

Murillo, L.A., Smith, P.H. \& Schall, J. (2010). Leading and learning literacy in three countries: Colombia, Mexico and the United States. Trabajo presentado en el $23^{\text {rd }}$ IRA World Congress on Reading. Leading and Learning in Literacy. Auckland, New Zealand.

Muter, V. \& Snowling, M. (1998). Concurrent and longitudinal predictors of reading: The role of metalinguistic and short-term memory skills. Reading Research Quarterly, 33 (3), 320-337. doi:10.1598/RRQ.33.3.4.

Nelson, K. (1996). Language in cognitive development: The emergence of the mediated mind. New York: Cambridge University Press.

Nelson, K. (2007). Young minds in social worlds: Experience, meaning, and memory. Cambridge, MA: Harvard University Press.
Newman, S. (2006). The knowledge gap: Implications for early education. En D.K. Dikinson \& S. Newman (Eds.), Handbook of early literacy development, Vol. 2. New York: Guilford.

Ninio, A. \& Bruner, J. (1978). The achievement and antecedents of labelling. Journal of Child Language, 5, 1-15. doi: 10.1017/S03050009 00001896.

Phillips, B., Clancy-Menchetti, J. \& Lonigan, C. (2008). Successful phonological awareness instruction with preschool children. Topics in Early Childhood Special Education, 28 (1), 3-17. doi: 10.1177/0271 121407313813.

Plana, M.D. (2012). Los niños en un mundo de eventos: Representaciones mentales y relatos [Children in a world of events: mental representations and storytelling]. Tesis Doctoral no publicada, Universidad Nacional de Córdoba. Córdoba. Argentina.

Read, C. (1971). Preschool children's knowledge of English phonology. Harvard Educational Review, 41, 1-34.

Reid, J.F. (1966). Learning to think about reading. Educational Research, 9, 56-62. doi: 10. 1080/0013188660090109.

Sautú, R. (1992). Teoría y medición del estatus ocupacional. Escalas ocupacionales objetivas y de prestigio [Theory and measure of employment status: Occupational scales]. Cuadernos del Instituto de Investigaciones, 10, Instituto de Investigaciones Gino Germani, Universidad de Buenos Aires.

Schneider, W., Kuespert, P., Roth, E. \& Vise, M. (1997). Short- and term effects of training phonological awareness in kindergarten: Evidence from two German studies. Journal of Experimental Child Psychology, 66(3), 311-340. doi: 10.1006/jecp.1997.2384.

Sinclair, A., Jarvella, R.J. \& Levelt, W.J.M. (1978). The child's conception of language. Berlin: Springer-Verlag Berlin Heidelberg. doi: 10.1007/978-3-642-67155-5_10. 
Snow, C.E., Porche, M.V., Tabors, P.O. \& Harris, S.S. (2007). Is literacy enough? Pathways to academic success for adolescents. Illinois: Brookes.

Spoelders, M. \& Van Damme, L. (1982). Early metalinguistic awareness and reading. Scientia Paedologica Experimentalis, 19(1), 119-129.

Stage, S.A. \& Wagner, R.K. (1992). Development of young children's phonological and orthographic knowledge as revealed by their spellings. Developmental Psychology, 28(2), 287-296. doi: 10.1037/0012-1649.28.2.287.

Stanovich, K.E. (1986). Matthew effects in reading: Some consequences of individual differences in the acquisition of literacy. Reading Research Quarterly, 21(4), 360407. doi: 10.1598/RRQ.21.4.1.

Storch, S.A. \& Whitehurst, G.J. (2002). Oral language and code-related precursors to reading: Evidence from a longitudinal structural model. Developmental Psychology, 38(6), 934947. doi: 10.1037//0012-1649.3 8.6.934.

Sulzby, E. (1983). Children's emergent abilities to read favorite story books. Evanston II: Northwestern University.

Sulzby, E. \& Teale, W. (1991). Emergent literacy. En R. Barr, M.L. Kamil, P.B. Mosenthal \& P.D. Pearson (Eds.), Handbook of reading research, Vol. 2 (pp. 727-757). New York: Longman.
Taylor, D. (1983). Family literacy. Exeter, NH: Heineman.

Treiman, R. (1991). Phonological awareness and its roles in learning to read and spell. En D. Sawyer \& B. Fox (Eds.), Phonological awareness in reading: The evolution of current perspectives (pp. 159-189). New York: Springer-Verlag.

Tunmer, W.E. \& Nesdale, A.R. (1985). Phonemic segmentation skill and beginning reading. Journal of Educational Psychology, 77(4), 417-427. doi: 10.1037//0022-0663. 77.4.417.

Vigotsky, L.S. (1979). El desarrollo de los procesos psicológicos superiores [The development of higher psychological processes]. Barcelona: Grijalbo.

Whitehurst, G.J. \& Lonigan, C.J. (1998). Child development and emergent literacy. Child Development, 69(3), 848-872. doi:10.2 307/ 1132208.

Yarden, D.B., Rowe, D.W., \& MacGillivray, L. (2011). Emergent Literacy: A matter (polyphony) of perspectives. En M.L. Kamil, P.D. Pearson, E. Birr Moje \& P.P. Afflerbaj (Eds.), Handbook of Reading Research, Vol. 3 (pp. 425-454). New Jersey: Earlbaum.

Ziegler, J.C. \& Goswami, U. (2005). Reading acquisition, developmental dyslexia, and skilled reading across languages: A psycholinguistic grain size theory. Psychological Bulletin, 131, 3-29. doi: 10.1 037/ 0033-2909.131.1.3.

Instituto de Lingüistica Facultad de Filosofía y Letras Universidad de Buenos Aires (UBA) Ciudad Autónoma de Buenos Aires República Argentina

Fecha de recepción: 27 de marzo de 2012 Fecha de aceptación: 2 de julio de 2012 